\title{
Spatial filtering using dielectric photonic crystals at beam-type excitation
}

Evrim Colak, Atilla Ozgur Cakmak, Andriy E. Serebryannikov, and Ekmel Ozbay

Citation: Journal of Applied Physics 108, 113106 (2010);

View online: https://doi.org/10.1063/1.3498810

View Table of Contents: http://aip.scitation.org/toc/jap/108/11

Published by the American Institute of Physics

\section{Articles you may be interested in}

Toward photonic crystal based spatial filters with wide angle ranges of total transmission

Applied Physics Letters 94, 181101 (2009); 10.1063/1.3127443

Spatial filtering with photonic crystals

Applied Physics Reviews 2, 011102 (2015); 10.1063/1.4907345

Super-collimation by axisymmetric photonic crystals

Applied Physics Letters 104, 221108 (2014); 10.1063/1.4881839

Intrinsic beam shaping mechanism in spatially modulated broad area semiconductor amplifiers

Applied Physics Letters 103, 132101 (2013); 10.1063/1.4821251

Spatial filtering using media with indefinite permittivity and permeability tensors

Applied Physics Letters 82, 2215 (2003); 10.1063/1.1562344

Self-collimating phenomena in photonic crystals

Applied Physics Letters 74, 1212 (1999); 10.1063/1.123502

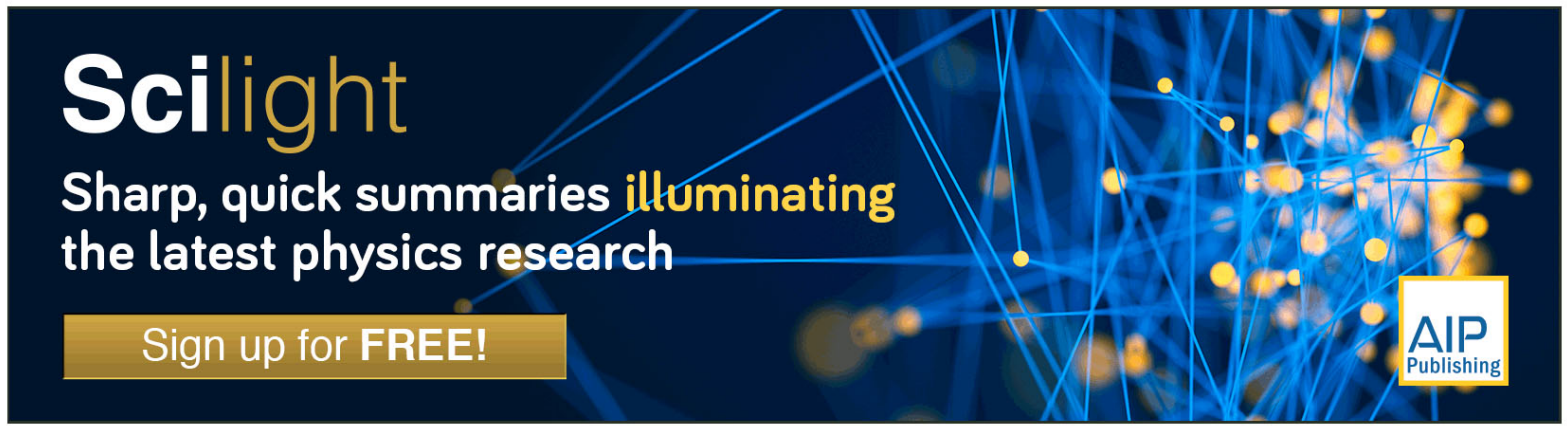




\title{
Spatial filtering using dielectric photonic crystals at beam-type excitation
}

\author{
Evrim Colak, ${ }^{1, a)}$ Atilla Ozgur Cakmak, ${ }^{1}$ Andriy E. Serebryannikov, ${ }^{2}$ and Ekmel Ozbay ${ }^{1}$ \\ ${ }^{1}$ Department of Electrical and Electronics Engineering, Department of Physics, Nanotechnology Research \\ Center, Bilkent University, 06800 Ankara, Turkey \\ ${ }^{2}$ Department of Electrical Engineering, E-3, Hamburg University of Technology, D-21071 Hamburg, \\ Germany
}

(Received 5 July 2010; accepted 1 September 2010; published online 6 December 2010)

\begin{abstract}
Spatial filtering is demonstrated at beam-type excitations by utilizing finite thickness slabs of two-dimensional dielectric photonic crystals (PCs) showing exotic Fabry-Perot resonances that are preserved over a wide range of variation of the incidence angle. Bandstop and dual-bandpass filtering effects are illustrated theoretically and the corresponding filters are validated in the microwave experiments by using square-lattice PCs. It is shown that the basic transmission features that were observed earlier for a plane-wave illumination are also recognizable at beam-type excitations. The proposed spatial filtering mechanism exhibits directional beaming. The desired widths and the locations of the passbands and stopbands are attainable in the angle domain with a proper choice of the operating frequency for the given excitation characteristics. () 2010 American Institute of Physics. [doi:10.1063/1.3498810]
\end{abstract}

\section{INTRODUCTION}

Spatial filters have been widely used for information processing and image enhancement in various ranges of electromagnetic spectrum. In particular, they were employed in the analysis of the spatial spectrum, the enhancement of the antenna directivity, radar data processing, aerial imaging, and distinguishing the incoming radiations with respect to the source location. The known theoretical and experimental implementations of the spatial filters involve those based on anisotropic media, ${ }^{1}$ multilayer stacks that are combined with a prism, ${ }^{2}$ resonant grating systems, ${ }^{3}$ interference patterns, ${ }^{4}$ metallic grids over a ground plane, ${ }^{5}$ two-dimensional (2D) photonic crystals (PCs) with ${ }^{6}$ and without ${ }^{7}$ defects, chirped $\mathrm{PCs}^{8}{ }^{8}$ as well as one-dimensional piecewise-homogeneous $\mathrm{PCs}^{9}$ and graded-index PCs. ${ }^{10}$ The coexisting spatial and frequency domain filtering has been studied in the context of controlling the laser radiation with the aid of the resonant grating based filters. ${ }^{11}$

Smoothing or, in other words, "cleaning" the beams radiated by the high-power lasers is an important application of the low-pass spatial filters. ${ }^{9,11}$ Filters of this type are considered to be comparably easier to realize since an anisotropiclike dispersion is not a prerequisite for the low-pass filters in contrast to the case of the high-pass and wide bandpass filters. ${ }^{1}$ It has been shown in Ref. 1 that wide adjacent ranges of transmittance, $T \leq 1$, and reflectance, $R=1$, can be obtained by using an anticutoff media, i.e., media with indefinite permittivity and permeability tensors. The simultaneous achievement of the high and nearly-constant transmittance within a wide range of the incidence angle, $\psi$, and a rather steep switching between the pass and stopbands constitute the general problems that are identified with the wide-band bandpass and bandstop filters.

In order to overcome these difficulties, an approach has

${ }^{a)}$ Electronic mail: ecolak@ee.bilkent.edu.tr. recently been offered for the plane-wave excitation, ${ }^{7}$ which is based on the use of the 2D dielectric PCs possessing nearly-flat isofrequency contours (IFCs) and yielding firstorder diffraction for large $\psi$. In the case of a square-lattice PC, IFCs that are localized around M point of the irreducible Brillouin zone are required for obtaining a bandpass spatial filter, whereas IFCs that are located simultaneously near $\Gamma$ and $\mathrm{M}$ points are needed for a bandstop spatial filter, at least if $\operatorname{sgn} \psi=$ const. It should be emphasized that the flatness of the IFCs is required for attaining high transmittance within an entire passband for the low-pass filtering, too. Therefore, a spatial filter based on a PC should be more advantageous than a performance that is based on the ultralow-index metallic wire medium. ${ }^{12}$

In the present paper, we numerically and experimentally examine the spatial filtering mechanism which is proposed in Ref. 7. However, the present study focuses on the realization of a PC based spatial filter operating under a beam-type excitation, instead of a hypothetical plane-wave illumination. The main goal is to demonstrate the principal features of the bandpass and bandstop spatial filters. First, we calculate the IFCs of the infinite PC. Second, the near-field results for a Gaussian-beam excitation are presented in order to demonstrate the collimation effects associated with the nature of the spatial filtering. Then, the far-field transmission results are investigated for a plane-wave excitation. They are exploited to determine the appropriate range of frequency and PC lattice parameter variation for the purposes of a microwave experiment. Conventional horn antennas are employed in the experiments. In turn, a wide Gaussian beam is introduced as the excitation in the finite difference time domain (FDTD) simulations. The experimental results are presented and discussed together with the numerical calculations. The directional beaming at the output of the PC is investigated. Finally, the basic features are summarized in the conclusion section. 


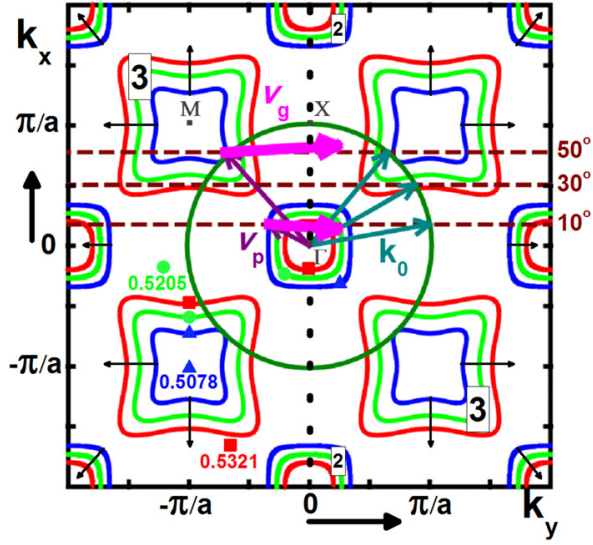

FIG. 1. (Color online) IFCs at $a / \lambda=0.5078$ (nearly square blue contours), $a / \lambda=0.5205$ (nearly square green contours) and $a / \lambda=0.5321$ (nearly square red contours). The boxed numbers on the IFCs, 2 and 3, correspond to the second and third lowest bands of the PC. Thin arrows show possible directions of group velocity, $\boldsymbol{v}_{g}$. The PC interface is along $\Gamma-\mathrm{X}$ direction (dotted black line). Air band (circle-shaped green contour around $\Gamma$ point), the incident wave vectors $\left(\boldsymbol{k}_{\boldsymbol{\theta}}\right.$, intermediately thick dark turquoise arrows), the phase velocities $\left(\boldsymbol{v}_{p}\right.$, intermediately thick purple arrows), the directions of $\boldsymbol{v}_{g}$ (thick pink arrows), and the construction lines (dashed wine-colored straight lines) at $\psi=10^{\circ}, 30^{\circ}$, and $50^{\circ}$ are shown for $a / \lambda=0.5078$. The IFCs are marked with solid geometrical shapes (blue triangle for $a / \lambda=0.5078$, green circle for $a / \lambda=0.5205$, and red square for $a / \lambda=0.5321$ ).

\section{THEORETICAL BACKGROUND}

Extensive numerical simulations have been carried out at plane-wave and Gaussian-beam excitations, in order to select a proper PC performance for experimental validation. Part of these results will be presented in the next section for convenience of comparison. Based on the obtained results, we decided in favor of the PC that is composed of circular alumina rods with a permittivity $\varepsilon_{r}=9.61$ and a diameter $d$ $=3.1 \mathrm{~mm}$. The rods are arranged in a square lattice with a lattice constant $a=7 \mathrm{~mm}$, so that $d / a=0.4429$. The structure contains eight rod layers. The interfaces are assumed to be parallel to the $\Gamma-\mathrm{X}$ direction. The selected parameters are quite close to those in Ref. 7. Accordingly, the basic dispersion and plane-wave transmission characteristics are expected to be similar to those in Figs. 2 and 4 from Ref. 7.

We made use of the plane-wave expansion method while calculating the dispersion for the corresponding infinite PC. The dispersion curves are given in Fig. 1 for three typical values of $a / \lambda$. It is assumed that the input interface is parallel to $k_{x}$ axis, while the $\mathrm{PC}$ is illuminated from the negative side of $k_{y}$ axis. According to Ref. 7, two nearly-flat IFCs must coexist at the same frequency in order to obtain a dualbandpass or a bandstop spatial filter at $\operatorname{sgn} \psi=$ const. The IFCs must be located in such a manner that the incident plane wave is coupled to a Floquet-Bloch (FB) wave of the PC only at large and small $\psi$. In turn, coupling to the FB waves at intermediate $\psi$ values is avoided by positioning one of the IFCs near $\Gamma$ point, while the other one is located near $M$ point. Such two IFCs might appear due to either two different FB waves or the same FB wave, depending on the choice of the PC lattice parameters. In Fig. 1, the IFCs localized around $\Gamma$ point belong to the second lowest band of the PC. They are shrinking down as the frequency is increased. Conversely, the IFCs that reside in the third lowest band are located in the vicinity of the M point. They expand outwards with the increasing frequency values. The shape of the IFC remains nearly the same, while the variations in frequency only lead to the modification of the IFC width. The IFCs near M point can lead to the appearance of the anticutoff-type passband.

In the ideal case, the IFC flatness should be an indicator of the possibility of achieving the total transmission, $T=1$, within a wide range of $\psi$-variation. In fact, if the operating frequency is chosen to be equal to that of an exotic FabryPerot resonance, one can stay in the close vicinity of this particular resonance in a broad span of $\psi$, in which the high transmission is sustained. This mechanism is distinguished from the classical $\psi$-dependent Fabry-Perot resonances which are known to be the common feature for the cases of $\mathrm{PC}$ bands with (approximately) isotropic dispersion. ${ }^{13}$ It is noteworthy that the same condition of the nearly-flat shape of the IFCs is required for the spatial filtering as for imaging and collimation. ${ }^{14-16}$ However, in contrast with the last two regimes, bandstop and bandpass spatial filtering is expected to be a result of a partial or, in other words, an angledependent collimation. Correspondingly, the location of the IFCs of the PC with respect to that of air should be different in case of spatial filtering. Indeed, in this case the collimation could appear with the plane-wave excitation only for the small and the large (excluding the intermediate) values of $\psi$, at which the incident wave is coupled to a FB wave. Otherwise, the total reflection should occur.

Specifically, the IFC for air may not be narrower than the PC IFCs, which are located near $\Gamma$ point. ${ }^{7}$ Then, the dualbandpass and bandstop regimes corresponding to these distinct frequency values are easily spotted in Fig. 1. The coupling mechanism has been explicitly examined for $a / \lambda$ $=0.5078$. The conservation of the tangential component of the incident wave vector at the PC interface dictates that the incident beam is coupled to a FB wave at $\psi=10^{\circ}$ and $\psi=50^{\circ}$, unlike the case of $\psi=30^{\circ}$. That is, $\boldsymbol{v}_{\boldsymbol{g}}$ and $\boldsymbol{v}_{\boldsymbol{p}}$ are shown only at $\psi=10^{\circ}$ and $\psi=50^{\circ}$, i.e., in those cases when the incident wave is coupled to a Floquet-Bloch wave. For $\psi=30^{\circ}$, there is no such coupling. This can be seen while inspecting the crossing points of the construction lines with the IFCs. Furthermore, the refracted beams (shown by pink thick arrows) propagate nearly parallel to the surface normals inside the PC as a result of the nearly-flat IFCs. The consequences of this collimation effect are going to be discussed in the next section. To sum up, the dispersion curves offer a preliminary estimation of the overall behavior of the PC based spatial filtering and provide us with the clues that comprise the coupling mechanisms for the particular incidence angles as well as the fundamental significance of the nearlyflat IFCs.

In order to demonstrate the connection of a $\psi$-domain passband with the appearance of the collimation regime, near-field patterns were examined at a Gaussian-beam excitation. The Gaussian wave has intentionally been chosen to be a beam with a width of $w=15 a$. Based on the near and far-field simulation results (not shown here), one can conclude that such a wide beam would enable us to make a fair analogy with the plane-wave illumination case. Figure 2 pre- 

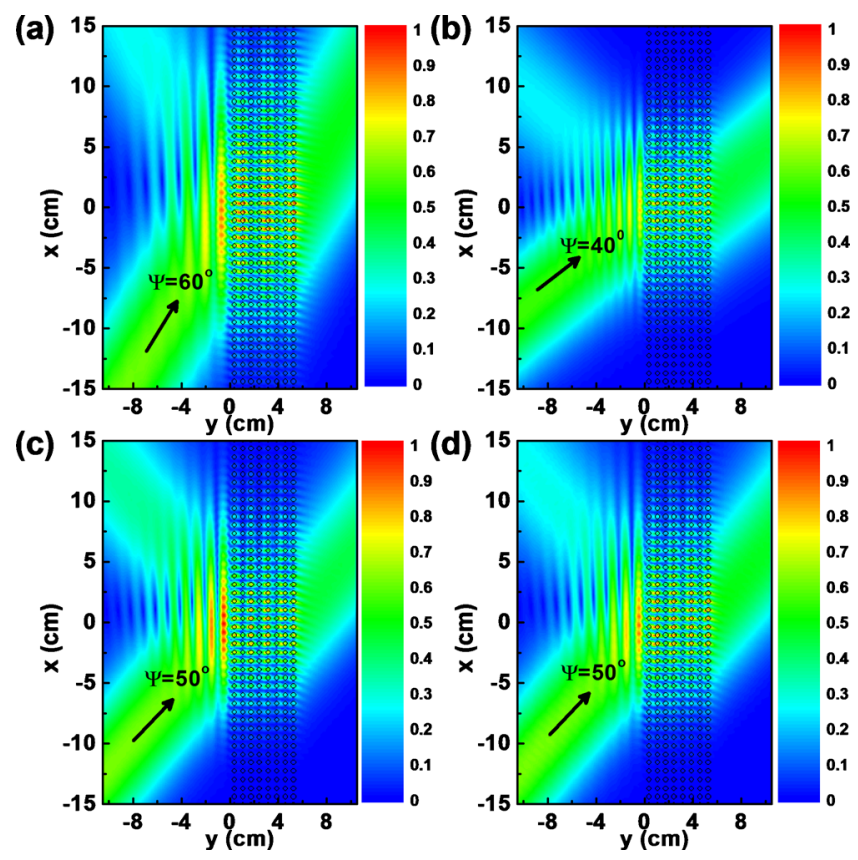

FIG. 2. (Color online) Electric field distributions at (a) $f=21.763 \mathrm{GHz}$, $a / \lambda=0.5078$, and $\psi=60^{\circ}$; (b) $f=22.804 \mathrm{GHz}, a / \lambda=0.5321$, and $\psi=40^{\circ}$; (c) $f=22.804 \mathrm{GHz}, a / \lambda=0.5321$, and $\psi=50^{\circ}$; and (d) $f=22.305 \mathrm{GHz}, a / \lambda$ $=0.5205$, and $\psi=50^{\circ}$.

sents the near-field results corresponding to the large- $\psi$ passband, which is associated with the IFCs located near M point. The field profiles are normalized individually with respect to the maximum electric field value at the particular frequency and incidence angle. One can see in all of the four plots of Fig. 2 that the beam inside the PC propagates in the direction which is nearly perpendicular to the interfaces, and leaves the PC while being neither blurred nor substantially shifted.

It is seen in Fig. 2 that a large portion of the incident beam energy can be transmitted through the PC, even at a rather large $\psi$, e.g., at $\psi=60^{\circ}$. However, the level of the reflections within the passband vastly depends on $\psi$. The plane-wave passband is flat and shows $T=1$ in the examples that are presented in Ref. 7. Yet, this would not guarantee the total transmission at a beam-type excitation because of the finite angular plane-wave spectrum of the beam. For a portion of the spectrum, coupling to the FB waves of the PC may take place, while for another one it may not. Furthermore, the effect of the PC interfaces can result in deviations from $T=1$ even if only the zero diffraction order is propagating, already for a plane-wave excitation. Thus, the cases that could be considered as the most appropriate ones for a planewave excitation are not necessarily the most suitable candidates for a nonplane-wave illumination.

In spite of this, the transmission spectra at a plane-wave excitation can still be useful for a proper choice of the operating frequency which should be equal or at least close to that of a Fabry-Perot-type resonance. ${ }^{17}$ In this respect, the plane-wave transmission characteristics have been calculated by using an integral equation technique. The examples are depicted in Fig. 3 for the frequencies which lie in the same frequency range as in Fig. 2. The stopband is well pro-

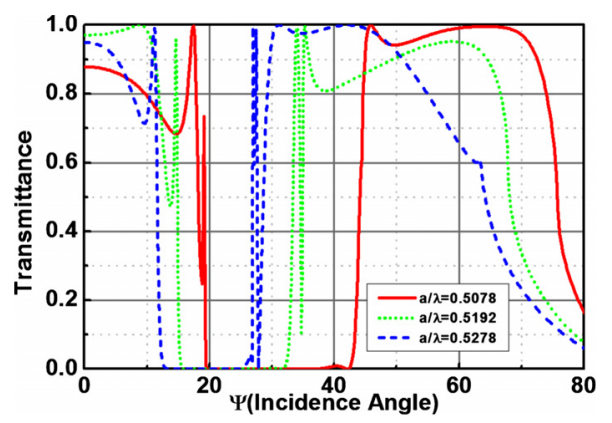

FIG. 3. (Color online) Zero order transmittance vs $\psi$ at $a / \lambda=0.5078$ (solid red line), $a / \lambda=0.5192$ (dotted green line), $a / \lambda=0.5278$ (dashed blue line). The transmission due to the first-order diffraction is non vanishing starting from $\psi=75.8^{\circ}, \psi=67^{\circ}$, and $\psi=63.5^{\circ}$, respectively.

nounced in all three cases. One can notice that the passband arising between $\psi=40^{\circ}$ and $80^{\circ}$ for $a / \lambda=0.5078$ is most appropriate for the purposes of bandpass filtering. In turn, the passband arising at $\psi<16^{\circ}$ for $a / \lambda=0.5192$ seems most appropriate for the low-pass filtering. At least for the materials available at microwave frequencies, obtaining a PC performance, for which $T \approx 1$ simultaneously within the small- $\psi$ and large- $\psi$ ranges, is still a challenging task. Hence, the cases shown in Fig. 3 as well as those with other similar parameters can be considered as quite appropriate for the purposes of the present study. The near-edge peaks lead to another problem to be solved. It should be noted that the passbands observed in Fig. 3 at small and large $\psi$ correspond to the second and third lowest PC bands, respectively, as it was already pointed out in the IFC diagrams (see Fig. 1). Besides, the large- $\psi$ passband is moving toward the smaller angles as the operating frequency is increased. The same feature is observed for the upper edge of the small- $\psi$ passband. These features are consistent with the predictions based on the IFCs.

\section{EXPERIMENTAL SETUP}

The angular dependence of the transmission through the PC was measured for a wide range of $\psi$-variation. The experimental setup contains Agilent two-port 8510C Network Analyzer and two conventional horn antennas with an operational frequency range from 18 to $25 \mathrm{GHz}$ (see Fig. 4). The $\mathrm{PC}$ is assembled as an array of $8 \times 100$ alumina rods with a length of $15.4 \mathrm{~cm}$. Even though a significant repertoire of the 3D PC studies has been accumulated for the extremely high

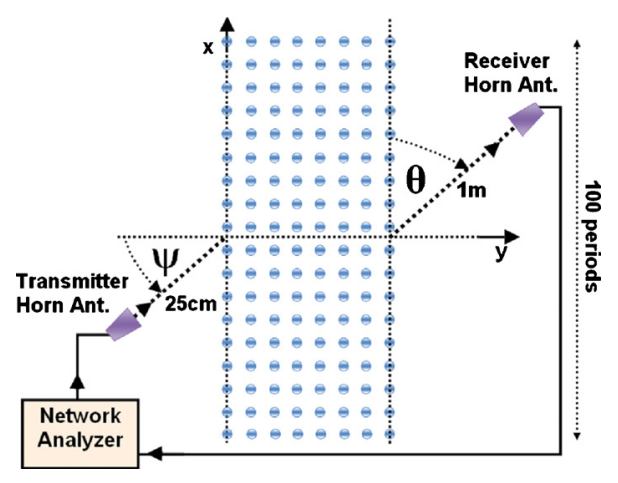

FIG. 4. (Color online) Schematic of the experimental setup. 
frequency regimes over the past decade, ${ }^{18,19}$ the electrical length of the rods is larger than $10 \lambda$ for every operational wavelength in our case, which safely allows our study to be considered as a $2 \mathrm{D}$ problem. The remaining parameters of the PC are the same as mentioned above. A horn antenna, placed $25 \mathrm{~cm}$ away from the PC, was used as the source for TE-polarized field (electric field vector is parallel to the rod axes). The value of $\psi$ has been varied from $0^{\circ}$ to $77^{\circ}$. The measurements of the angular distribution of the transmission coefficient were carried out by employing another horn antenna as a receiver, which has scanned the semicircular path so that the receiver was kept $1 \mathrm{~m}$ away from the center of the output interface of the PC, as shown in Fig. 4.

The used antennas are standard reciprocal pyramidal horn antennas which have a calculated directivity $D$ $\approx 13.98 \mathrm{~dB}$ and a half power beamwidth, $\mathrm{HPBW} \approx 18^{\circ}$, at $\lambda=1.43 \mathrm{~cm}(f=21 \mathrm{GHz})$ for the H-plane $(x-y$ plane in Fig. 4). The antenna directivity and thereby the radiation patterns differ within the frequency band of operation. The radiated fields of the horn antenna are expressed with rather complicated Fresnel Integrals. ${ }^{20}$ In turn, the radius of curvature of the wavefronts at Gaussian-beam excitation in the simulations was evaluated to be $R=18.38 \mathrm{~m}$ at $\lambda=1.43 \mathrm{~cm}$ at the $\mathrm{PC}$ input interface. Such a high value ensures that the nearlyplanar wavefronts do reach the PC interface at Gaussianbeam illumination. The Gaussian source that was employed in the simulations has been a bridge connecting the gap in between the hypothetical plane-wave illumination and the real life applications. The area of the transmitter antenna aperture was $4.5 \times 6.4 \mathrm{~cm}^{2}$, so that the Fresnel number $F$ $=A_{\max }^{2} / L \lambda \approx 1.71$ at $\lambda=1.43 \mathrm{~cm}$, where $A_{\max }=7.82 \mathrm{~cm}$ and $L=25 \mathrm{~cm}^{21}$ Hence, the input interface of the PC is located at the Fresnel zone of the transmitter antenna and the incident wavefronts keep the nonplane-wave features in the experiments.

The receiver antenna was of the same size as the transmitter one. A refocusing has not been applied to the transmitted field. The receiver antenna, which was situated $1 \mathrm{~m}$ apart, is close to the boundary of its Fraunhofer zone. In the case without the $\mathrm{PC}$, the experimental radiation patterns and the HPBW attain values which are very close to the calculated free-space far-field results for the pyramidal horn antenna. ${ }^{20}$ Likewise, the transmitter antenna could also be positioned $1 \mathrm{~m}$ away from the PC input interface in order to reshape the incident beam wavefronts but this would in turn cause unwanted diffractions from the shorter sides and corners of the PC. In fact, the PC acts as an array antenna with a large effective aperture and contributes to the shaping of the outgoing radiation at the output side..$^{22,23}$ Then, we are compelled to stay in the near field for several meters of radial measurement distances. In summary, we are bound to put the horn antennas in the near field regions on both sides of the $\mathrm{PC}$ at the operating frequencies throughout the measurements since some experimental difficulties (signal attenuation, wave broadening, parasitic diffractions) can appear for larger distances. In contrast to the Gaussian-beam excitation case, the incident beam arrives at the PC interface with nonplanar wavefronts, which constitutes the main difference between the antenna and Gaussian-beam excitations. Neverthe- (a)

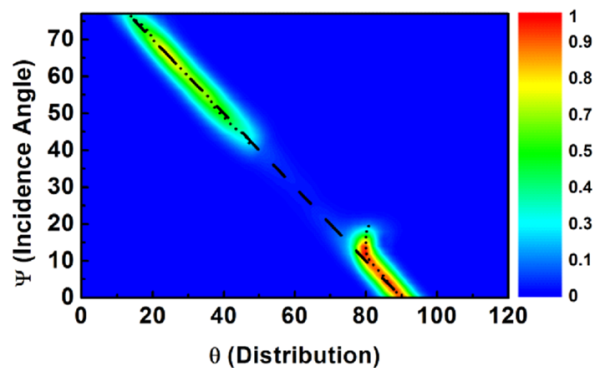

(b)

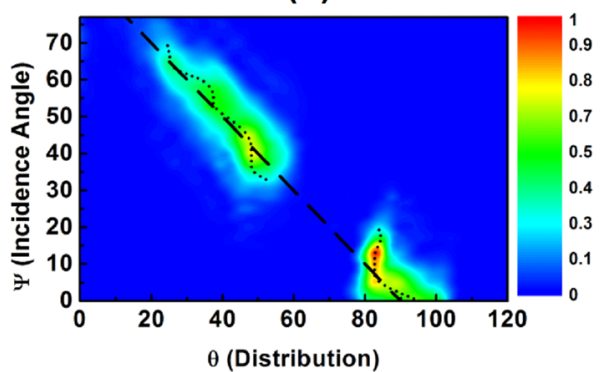

FIG. 5. (Color online) Transmittance on the $(\theta, \psi)$-plane at $f$ $=21.763 \mathrm{GHz}(a / \lambda=0.5078)$ for (a) Gaussian-beam excitation, FDTD simulations and (b) horn-antenna excitation, experiment.

less, in Sec. IV, we are going to demonstrate that the basic principles of the spatial filtering are still achievable under these conditions.

\section{RESULTS AND DISCUSSION}

Results at several frequencies are presented simultaneously for the wide Gaussian beam and the horn-antenna excitation, so that one can clearly see the effect of changing the type of the excitation. These results contain radiation patterns and maps of transmittance on the $(\theta, \psi)$-plane, where $\theta$ is the observation angle. The transmitted intensities are normalized with respect to the maximum attainable value at each frequency. Figure 5 presents the maps of transmittance for the same frequency value as in Fig. 2(a) and in one of the cases in Fig. 3. Two $\psi$-domain passbands are observed, but their boundaries are now blurred due to the finite angular spectrum as opposed to the plane-wave case. Stopbands appear in both $\theta$ - and $\psi$-domains. There are no alternating minima and maxima within each passband, which further support that the spatial filtering mechanism is realized by staying in a rather close vicinity of the same Fabry-Perottype resonance.

The location of the stopbands in Fig. 5 can be quite well predicted using the plane-wave results. Nonetheless, the angular spectrum of the incident beam launched from the horn antenna is apparently wider, leading to broader $\theta$-ranges of transmission at a fixed $\psi$. A substantial transmission happens to be detected in the experiments for the angle range $35^{\circ}$ $<\psi<40^{\circ}$. The transmission within this particular range was either vanishing or insignificant at the Gaussian-beam and plane-wave illuminations. This might occur since the nonplane-wave features of the antenna illumination become more important, leading to the stronger contribution of the angular spectrum components which correspond to the stop- 
(a)

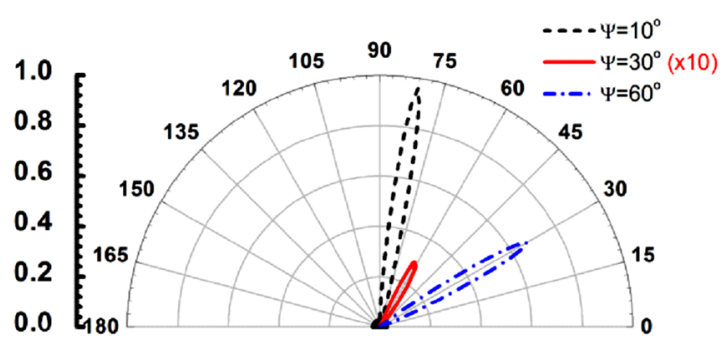

(b)

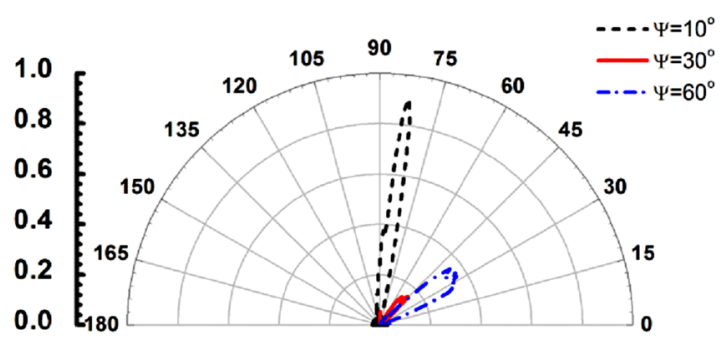

FIG. 6. (Color online) Radiation patterns for Fig. 5 at three typical values of $\psi: \psi=10^{\circ}$ (dashed black line), $\psi=30^{\circ}$ (solid red line), and $\psi=60^{\circ}$ (dashdotted blue line). (a) Gaussian-beam excitation, FDTD simulations, (b) horn antenna excitation, experiment.

band at the plane-wave excitation. Moreover, the upper boundary of the second passband is observed at a smaller $\psi$ for the antenna case. Here, a bigger part of the angular spectrum should correspond to the values of $\psi$ at which the contribution of the zero order to the transmission in the planewave case is weaker.

In Fig. 5 and in all other similar figures, the dashed lines are expressed with the equation $\theta=\pi / 2-\psi$ whereas the dotted lines designate the maximum value of the transmitted intensity at every $\psi$. When these two lines coincide, the collimation takes place. As a result, the dominant components in the angular spectrum of the incident wave (i.e., the planewave incident at angle $\psi$ and its neighbors) propagate through the PC in the direction which is perpendicular to its interfaces. Among the various causes of deviation of the dotted lines from the dashed ones, we should refer to the non ideally flat IFCs and to that a significant part of the wide angular spectrum can be out of resonance, so that different plane-wave components contribute to the transmission with different weights. The latter cause is especially important for the horn-antenna excitation. In spite of this, the collimationlike behavior is still evident in the second passband, in accordance with our earlier claims about Fig. 2.

However, when we inspect the dotted lines in Fig. 5 at $10^{\circ}<\psi<20^{\circ}$, we notice the signatures of negative refraction. ${ }^{24,25}$ In fact, the deviation of the dotted lines toward larger $\psi$ at a fixed $\theta$ suggests negative refraction, which is in agreement with the dispersion features (see Fig. 1). It is noteworthy that this effect can be enhanced for the hornantenna excitation owing to a wider angular spectrum of the incident wave. Moreover, a collection of the radiation patterns at $a / \lambda=0.5078$, which correspond to the $\psi$-domain first passband (smaller $\psi$ ), stopband (intermediate $\psi$ ), and second passband (larger $\psi$ ) in Fig. 5, are presented in Fig. 6. Radia-

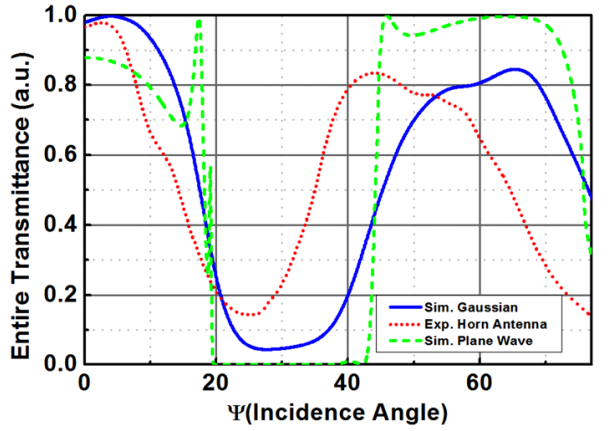

FIG. 7. (Color online) Entire transmittance for different illuminations at $f$ $=21.763 \mathrm{GHz}(a / \lambda=0.5078)$ for Gaussian-beam excitation (solid blue line), horn-antenna excitation in the experiments (dotted red line), and plane-wave excitation (dashed green line).

tion patterns simply consist of single slices that are extracted from the maps of transmittance at a desired incidence angle.

Figure 7 portrays the entire transmittance $T_{\Sigma}$ that is acquired by numerically integrating the transmitted intensity values over all observation angles in Fig. 5(a) (solid blue line) and Fig. 5(b) (dotted red line), respectively. For the comparison, the plane-wave excitation results from Fig. 3 are also plotted in Fig. 7 (dashed green line). Despite the broadening of the angular spectrum, the basic features of the spatial filtering, which were detected for the plane-wave excitation, still manifest for nonplane-wave excitations. In particular, two passbands of $T_{\Sigma}>0.5$ ( $3 \mathrm{~dB}$ point) do occur.

The locations, the widths, and the transmission levels of the passbands can be altered by adjusting the operating frequency. The IFCs in Fig. 1 remind us that an increment in the frequency results in simultaneously narrowing the first passband and widening the second passbands. In Fig. 8, an example of a map of transmittance is shown at a higher frequency, which corresponds to $a / \lambda=0.5321$. Now, the

\section{(a)}

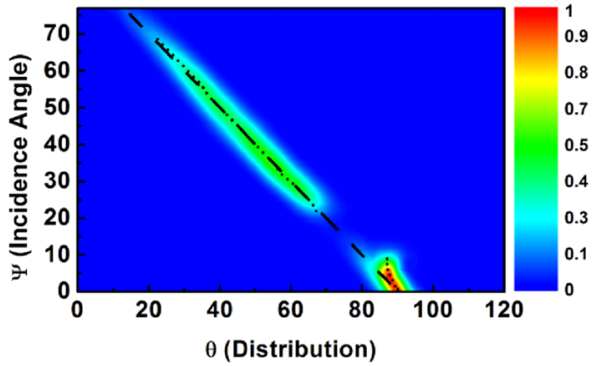

(b)

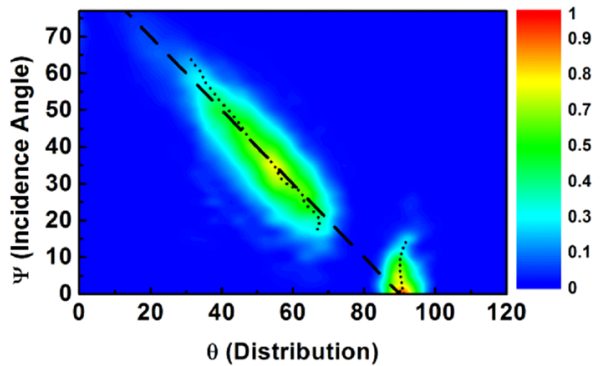

FIG. 8. (Color online) Transmittance on the $(\theta, \psi)$-plane at $f$ $=22.804 \mathrm{GHz}(a / \lambda=0.5321)$ for (a) Gaussian-beam excitation, FDTD simulations, and (b) horn-antenna excitation, experiment. 
(a)

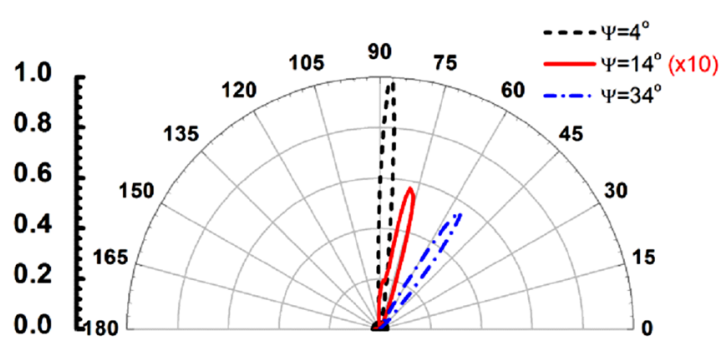

(b)

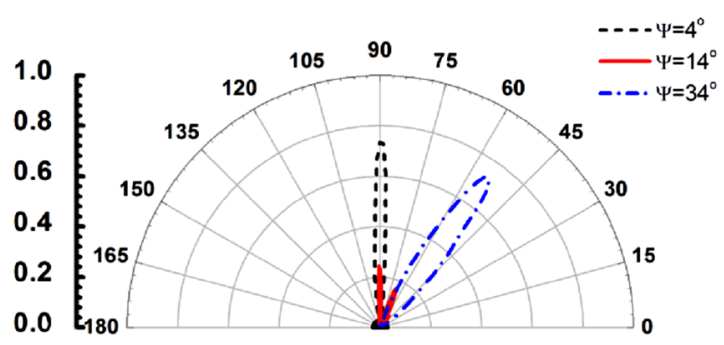

FIG. 9. (Color online) Radiation patterns for Fig. 8 at three typical values of $\psi: \psi=4^{\circ}$ (dashed black line), $\psi=14^{\circ}$ (solid red line), and $\psi=34^{\circ}$ (dashdotted blue line). (a) Gaussian-beam excitation, FDTD simulations, (b) horn-antenna excitation, experiment.

$\psi$-domain stopband is observed between the first and the second passbands only in the case of the Gaussian-beam illumination. At the same time, the $\theta$-domain stopband is maintained for both cases of the nonplane-wave illumination. It should also be noted that the collimation of the incoming wave is reinforced in the second passband [compare Figs. 5(b) and 8(b)]. For the first passband, the signature of the negative refraction starts appearing in Fig. 8(b) already at $\psi \approx 0$. Figure 9 shows the radiation patterns for the three selected values of $\psi$. The slices are again particularly picked among the first passband (small $\psi$ ), stopband (intermediate $\psi)$, and second passband (large $\psi$ ) in Fig. 8(a). One can notice the deviation of the slice in Fig. 9(b) toward larger $\theta$ as compared to Fig. 9(a). This corresponds to the negative refraction arising at small $\psi$ due to the contribution of the part of the angular spectrum that is associated with the plane waves incident at larger angles.

Figure 10 presents the map of transmittance at a frequency value that is intermediate between those in Figs. 5 and 8. Here, it corresponds to $a / \lambda=0.5192$, which is the same as in one of the cases in Fig. 3. The features observed in Fig. 10(b) are comparable to those in Figs. 5(b) and 8(b). The effect of spatial filtering is recognizable. However, now the lower boundary of the second $\psi$-domain passband and the upper boundary of the first passband nearly coincide. Thus, the stopband in $\psi$-domain tends to vanish. The radiation patterns for the three selected values of $\psi$ are presented in Fig. 11. The measured maximal values of $T$ at $\psi=10^{\circ}$ and $\psi=40^{\circ}$ in Fig. 11(b) are close to each other. This is fairly the result of a special choice of $\psi$-values. The maximal values of $T$ can vary with $\psi$ rather strongly inside the first and second passbands (both for the cases that are shown and not shown), especially at the horn-antenna excitation. Furthermore, the first and the second passbands have different maximal values (a)

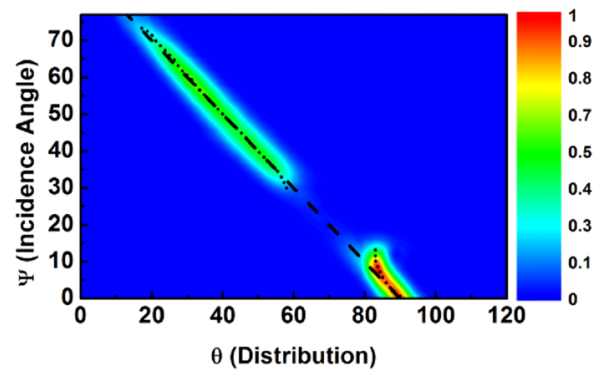

(b)

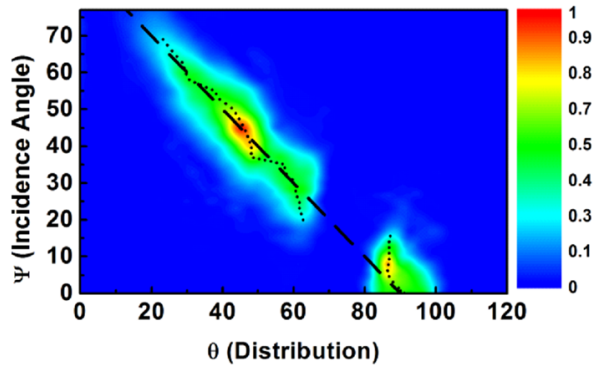

FIG. 10. (Color online) Transmittance on the $(\theta, \psi)$-plane at $f$ $=22.252 \mathrm{GHz}(a / \lambda=0.5192)$ for (a) Gaussian-beam excitation, FDTD simulations and (b) horn-antenna excitation, experiment.

of $T^{\prime}=\max T(\psi)$. These performance issues differ from the behavior of an ideal dual-bandpass filter. Yet, there are several effects which can lead to these discrepancies. First of all, one should mention the possibly different Fabry-Perot resonance frequencies for the two bands. The contribution of the plane-wave components, which are out of resonance, and the sensitivity of the transmittance values to a relatively weak

(a)

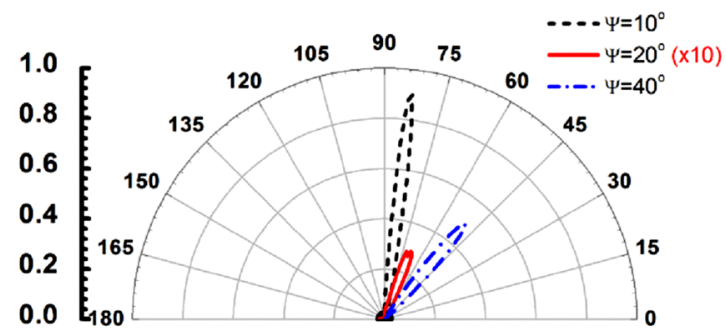

(b)

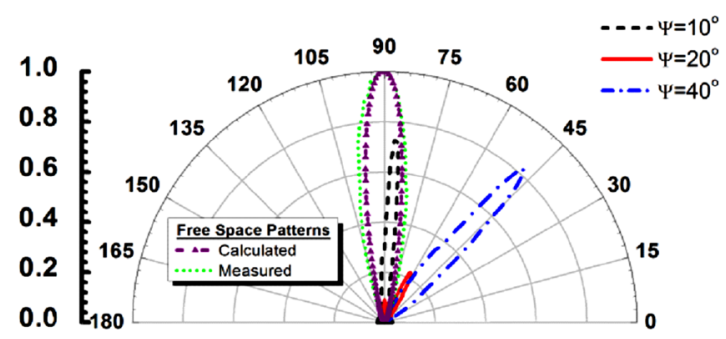

FIG. 11. (Color online) Radiation patterns for Fig. 10 at three typical values of $\psi: \psi=10^{\circ}$ (dashed black line), $\psi=20^{\circ}$ (solid red line), and $\psi=40^{\circ}$ (dashdotted blue line). (a) Gaussian-beam excitation, FDTD simulations, (b) horn-antenna excitation, experiment. The calculated (dotted green line) and measured (dashed purple line with triangles) free-space radiation patterns of the horn antenna at $\psi=0^{\circ}$ and $f=22.252 \mathrm{GHz}(a / \lambda=0.5192)$ are also plotted. 
variation in $\psi$ in the vicinity of the resonance frequencies can be listed as additional reasons that are responsible for the deviations from an ideal filter performance. The diffractions at the PC interfaces also affect the transmission, while being dependent on the excitation type. These effects should be necessarily taken into account while designing and experimentally testing a spatial filter for an optimal performance. As it can be deduced from the relevant figures, a $\theta$-domain stopband filtering is easier to succeed than a simultaneous stopband in the $\psi$ - and $\theta$-domains. Consequently, the frequency of operation should be downshifted if a wider $\psi$-domain stopband is aimed.

Lastly, the spatial filtering is accompanied with directional beaming, which can be clearly inferred from the given radiation patterns. It is known that directional beaming can be obtained by utilizing the corrugations at the interface layers of the PCs by exploiting the surface waves. ${ }^{26-29}$ Alternatively, the directional beaming can be achieved in PC with self-collimation abilities. ${ }^{30}$ Similarly, a collimation-type regime is observed together with directional beaming in the present study. The HPBW values stay lower than $20^{\circ}$ in the $\mathrm{H}$-plane throughout the passbands for all cases, both in the simulations and experiments. The near-edge and defect-mode regimes in the PCs are also known to have the capability to further improve the directivity factors of the incorporated emission sources. ${ }^{31}$ Likewise, a significant angular confinement of the emitted beam can be spotted in our measured radiation patterns. The used antennas have a $\mathrm{HPBW} \approx 20^{\circ}$ at $a / \lambda=0.5192$ for the free-space measurements [see Fig. 11(b)]. Conversely, the spatial filtering mechanism promises a $\mathrm{HPBW} \approx 11^{\circ}$ at $a / \lambda=0.5192$ and $\psi=40^{\circ}$ in the second passband. The angular confinement of the beam is even more improved at smaller observation angles which correspond to the first passband with negative refraction. The radiation patterns in Fig. 11(b) indicate that a $\mathrm{HPBW} \approx 6^{\circ}$ can be achieved at $a / \lambda=0.5192$ and $\psi=10^{\circ}$. Such narrow beams are attainable in the first passband for the other studied cases, too [see Figs. 6(b) and 9(b)].

\section{CONCLUSION}

To summarize, dual-bandpass and bandstop spatial filters can be obtained in classical dielectric PCs by proper adjustments of the lattice parameters and the frequency range. The fundamental features of the spatial filtering have been demonstrated both theoretically and experimentally. The obtained results validate the possibility of utilizing the spatial filtering mechanism, which has recently been suggested for the planewave incidence, at nonplane-wave excitations like a wide Gaussian beam and a Fresnel zone horn antenna. The extensive numerical and experimental studies have been performed for the former and the latter, respectively.

For the plane-wave excitation, the possibility of a wideband spatial filtering exists due to the specific Fabry-Perottype resonances which are nearly independent on the angle of incidence. As a result, one can stay in the close vicinity of the same resonance within a wide angle-domain passband while the frequency is fixed. In fact, the utilized mechanism can be interpreted in terms of the angle-dependent collima- tion which appears in the PC with nearly-flat IFCs and provides a coupling of the incident wave to a FB wave in the PC within a limited range of the incidence angles. In case of a nonplane-wave excitation, the deviations from the planewave characteristics occur owing to the finite angular spectrum of the incoming waves. However, the basic features do remain. The near-field simulation results clearly put forward the existence of the collimation regime at a Gaussian-beam excitation. The spatial filtering in the incidence and observation angle domains has been discussed in detail under the light of the numerical and experimental results obtained for the nonplane-wave excitations. The locations and the transmission levels of the passbands are examined as a function of frequency. The plane-wave transmission results and the dispersion results for the corresponding infinite PC usually provide the proper initial estimates for the passband locations at a nonplane-wave excitation. It is shown that the spatial filters can be engineered so that a stopband appears either simultaneously in the incidence and observation angle domains, or only in the observation angle domain. Finally, the improvement of the angular confinement of the outgoing beams is discussed with the aid of the measured radiation patterns.

Future works will include the design of the advanced single- and dual-bandpass spatial filters, as well as the theoretical studies attending the effect of the angular plane-wave spectrum and the curvature of the incident wavefronts, which have been beyond the main scope of the present paper. The proposed spatial filtering mechanism is demonstrated to produce highly directional beams with improved angular confinement. It is expected that an experimental performance of the spatial filter with appropriate transmission and angular selectivity characteristics can be designed at the optical frequencies, too. The adaptation of the proposed filter in the optical domain also relieves the restrictions on cooperating additional lenses and similar other optical devices for beam shaping and guiding, since the filtering mechanism is primarily handled by a single PC with a finite thickness. Eventually, it is anticipated that such a filter will have a wide range of applications in the optical communication systems as long as some form of an angular selectivity is needed.

\section{ACKNOWLEDGMENTS}

This work has been supported by the European Union under the Projects EU-PHOME, EU-ECONAM, and TUBITAK under the Project Nos. 107A004 and 107A012. A.S. thanks the Deutsche Forschungsgemeinschaft for support of this work under the Project No. SE1409.2-1.

${ }^{1}$ D. Schurig and D. R. Smith, Appl. Phys. Lett. 82, 2215 (2003).

${ }^{2}$ I. Moreno, J. J. Araiza, and M. Avendano-Alejo, Opt. Lett. 30, 914 (2005).

${ }^{3}$ A. Sentenac and A.-L. Fehrembach, J. Opt. Soc. Am. A 22, 475 (2005).

${ }^{4}$ L. Dettwiller and P. Chavel, J. Opt. Soc. Am. A 1, 18 (1984).

${ }^{5}$ O. F. Siddiqui and G. Eleftheriades, J. Appl. Phys. 99, 083102 (2006).

${ }^{6}$ Y. J. Lee, J. Yeo, R. Mittra, and W. S. Park, IEEE Trans. Antennas Propag. 53, 224 (2005).

${ }^{7}$ A. E. Serebryannikov, A. Y. Petrov, and E. Ozbay, Appl. Phys. Lett. 94, 181101 (2009).

${ }^{8}$ K. Staliunas and V. J. Sanchez-Morcillo, Phys. Rev. A 79, 053807 (2009). ${ }^{9}$ Z. Luo, Z. Tang, Y. Xiang, H. Luo, and S. Wen, Appl. Phys. B: Lasers Opt. 94, 641 (2009). 
${ }^{10}$ P. V. Usik, A. E. Serebryannikov, and E. Ozbay, Opt. Commun. 282, 4490 (2009).

${ }^{11}$ R. Rabady and I. Avrutsky, Opt. Lett. 29, 605 (2004).

${ }^{12}$ B. T. Schwartz and R. Piestun, J. Opt. Soc. Am. B 20, 2448 (2003).

${ }^{13}$ K. Sakoda, Optical Properties of Photonic Crystals (Springer-Verlag, Berlin, 2005).

${ }^{14}$ P. A. Belov, C. R. Simovski, and P. Ikonen, Phys. Rev. B 71, 193105 (2005).

${ }^{15}$ C. Luo, S. G. Johnson, J. D. Joannopoulos, and J. B. Pendry, Phys. Rev. B 65, 201104 (2002).

${ }^{16}$ T. P. White, C. M. de Sterke, R. C. McPhedran, and L. C. Botten, Appl. Phys. Lett. 87, 111107 (2005).

${ }^{17}$ B. Temelkuran, E. Ozbay, J. P. Kavanaugh, G. Tuttle, and K. M. Ho, Appl. Phys. Lett. 72, 2376 (1998).

${ }^{18}$ E. Özbay, E. Michel, G. Tuttle, R. Biswas, K. M. Ho, J. Bostak, and D. M. Bloom, Appl. Phys. Lett. 65, 1617 (1994).

${ }^{19}$ E. Özbay, G. Tuttle, J. S. McCalmont, M. Sigalas, R. Biswas, C. M. Soukoulis, and K. M. Ho, Appl. Phys. Lett. 67, 1969 (1995).

${ }^{20}$ C. A. Balanis, Antenna Theory: Analysis and Design (Wiley, New York,
2005).

${ }^{21}$ B. E. A. Saleh and M. C. Teich, Fundamentals of Photonics (Wiley, New York, 1991).

${ }^{22}$ E. Colak, H. Caglayan, A. O. Cakmak, A. D. Villa, F. Capolino, and E. Ozbay, Opt. Express 17, 9879 (2009).

${ }^{23}$ I. Bulu, H. Caglayan, and E. Ozbay, Opt. Lett. 30, 3078 (2005).

${ }^{24}$ E. Cubukcu, K. Aydin, E. Ozbay, S. Foteinopoulou, and C. M. Soukoulis, Nature (London) 423, 604 (2003).

${ }^{25}$ E. Cubukcu, K. Aydin, E. Ozbay, S. Foteinopolou, and C. M. Soukoulis, Phys. Rev. Lett. 91, 207401 (2003).

${ }^{26}$ M. S. Kumar, S. Menabde, S. Yu, and N. Park, J. Opt. Soc. Am. B 27, 343 (2010).

${ }^{27}$ S. K. Morrison and Y. S. Kivshar, Appl. Phys. Lett. 86, 081110 (2005).

${ }^{28}$ E. Moreno, F. J. Garcia-Vidal, and L. Martin-Moreno, Phys. Rev. B 69, 121402 (2004)

${ }^{29}$ P. Kramper, M. Agio, C. M. Soukoulis, A. Birner, F. Müller, R. B. Wehrspohn, U. Gösele, and V. Sandoghdar, Phys. Rev. Lett. 92, 113903 (2004). ${ }^{30}$ D. Tang, L. Chen, and W. Ding, Appl. Phys. Lett. 89, 131120 (2006).

${ }^{31}$ H. Caglayan, I. Bulu, and E. Ozbay, Opt. Express 13, 7645 (2005). 